\title{
Reviewers Index
}

(The reviewers who completed the first peer-review for manuscripts submitted during Nov 1, 2017-Oct 31, 2018.)

Annals of Vascular Diseases do thank all the reviewers for their strong support and cooperation.

A

Adachi, Koichi

Akasaka, Kazumi

Akashi, Hidetoshi

Akihiro, Yoshitake

Al-Salman, Mussaad

Altarazi, Louay

Alzahrani, Hasan Ali

Ando, Motomi

Aoyagi, Shigeaki

Azuma, Nobuyoshi

B

Bashar, Abul Hasan

Muhammad

Bessho, Ryuzo

C

Cheng, Stephen

\section{D}

Deguchi, Juno

\section{F}

Fletcher, John

Fukuda, Hirotsugu

Fukuda, Ikuo

Fukui, Kozo

\section{G}

Gottsäter, Anders

\section{H}

Hamamoto, Masaki

Hanaoka, Kazuo

Handa, Nobuhiro

Harada, Hirohisa

Hashizume, Kenichi

Hattori, Tsutomu

Hayashida, Naoki

Higashi, Yukihito

Hirokawa, Masayuki

Hirose, Hitoshi

Hoshina, Katsuyuki

Hosoi, Yutaka

Huh, Seung

\section{I}

Imoto, Yutaka

Inaba, Masashi

Inoue, Yoshinori
Ishibashi, Hiroyuki

Ishikawa, Tetsuya

Ito, Hiroyuki

Iwata, Hirohide

Iyori, Keiji

Izumi, Yuichi

\section{J}

Jibiki, Masatoshi

Jin, Hyun Joh

\section{K}

Kadohama, Takayuki

Kamal, Dhafer

Kasashima, Fuminori

Katsumata, Takahiro

Kawabori, Masashi

Kawaharada, Nobuyoshi

Kichikawa, Kimihiko

Kim, Young-Wook

Kitagawa, Takeshi

Kitano, Ikurou

Kobayashi, Masayoshi

Koitabashi, Norimichi

Koizumi, Jun

Kokubo, Taku

Komai, Hiroyoshi

Kritayakirana, Kritaya

Kudo, Toshifumi

Kuma, Sosei

Kumakura, Hisao

Kuniyoshi, Yukio

Kurabayashi, Masahiko

Kurimoto, Yoshihiko

Kutsukata, Noriyoshi

Kwon, Tae-Won

\section{L}

Laredo, James

\section{M}

Maeda, Hideaki

Maemura, Koji

Maruyama, Yoshiaki

Masaki, Hisao

Matsubara, Shinobu

Matsuda, Hitoshi

Matsumoto, Takuya

Matsushita, Masahiro

Midorikawa, Hirofumi

Minatoya, Kenji
Misawa, Yoshio

Miura, Hiroshi

Miyairi, Takeshi

Miyata, Tetsuro

Mo, Makoto

Moon, In Sung

Morishita, Kyofumi

Morota, Tetsuro

Motomura, Noboru

Murakami, Atsubumi

\section{$\mathbf{N}$}

Nakajima, Tadashi

Nakamura, Takashi

Nakazawa, Tatsu

Ninomiya, Junichi

Nishibe, Toshiya

Nishigami, Kazuhiro

Nishikimi, Naomichi

Nishimura, Kengo

Nishimura, Motonobu

Nishimura, Yoshiharu

Nunokawa, Masao

\section{O}

Obara, Hideaki

Ogino, Hitoshi

Ohki, Takao

Ohta, Takashi

Ohtake, Hiroshi

Okazaki, Jin

Okita, Yutaka

Orihashi, Kazumasa

Otani, Norifumi

P

Paocharoen, Veeraya

\section{S}

Saito, Yukihiro

Sakuda, Hitoshi

Sasaguri, Shiro

Sato, Osamu

Satokawa, Hirono

Seto, Tatsuichiro

Shibuya, Takashi

Shigematsu, Kunihiro

Shih, Chun Che

Shindo, Shunya

Sugano, Norihide

Sugimoto, Ikuo
Sugimoto, Takaki

Sumi, Makoto

Suzuki, Shinichi

T

Taguchi, Shinichi

Takagi, Gen

Takahashi, Toshiki

Takano, Hiroshi

Takase, Kei

Takayama, Toshio

Takayama, Yutaka

Toya, Naoki

$\mathbf{U}$

Ueyama, Keishi

Urayama, Hiroshi

W

Wada, Hideichi

Wang, Jinsong

Y

Yamada, Norikazu

Yamaguchi, Atsushi

Yamaki, Takashi

Yamamoto, Hiroshi

Yamamoto, Kota

Yamamoto, Naoto

Yasugi, Takumi

Yiu, Wai-ki

Yoshida, Hiroki

Yoshimura, Koichi

Yoshizumi, Masao

Yunoki, Yasuhiro

Yuri, Koichi

$\mathbf{Z}$

Zempo, Nobuya

(cc) BY-NC-SA (02018 The Editorial Committee of Annals of Vascular Diseases. This article is distributed under the terms of the Creative Commons Attribution License, which permits use, distribution, and reproduction in any medium, provided the credit of the original work, a link to the license, and indication of any change are properly given, and the original work is not used for commercial purposes. Remixed or transformed contributions must be distributed under the same license as the original. 